\title{
Effects of eight neuropsychiatric copy number variants on
} human brain structure

Claudia Modenato $\mathbb{D}^{1,74}$, Kuldeep Kumar $\mathbb{D}^{2,74}$, Clara Moreau ${ }^{2}$, Sandra Martin-Brevet ${ }^{1}$, Guillaume Huguet $\mathbb{D}^{2}$, Catherine Schramm ${ }^{2}$, Martineau Jean-Louis ${ }^{2}$, Charles-Olivier Martin², Nadine Youniss, Petra Tamer ${ }^{2}$, Elise Douard², Fanny Thébault-Dagher ${ }^{2}$, Valérie Côté ${ }^{2}$, Audrey-Rose Charlebois' ${ }^{2}$, Florence Deguire ${ }^{2}$, Anne M. Maillard ${ }^{3}$, Borja Rodriguez-Herreros ${ }^{3}$, Aurèlie Pain ${ }^{3}$, Sonia Richetin ${ }^{3}, 16 \mathrm{p} 11.2$ European Consortium*, Simons Searchlight Consortium*, Lester Melie-Garcia (iD ${ }^{4}$, Leila Kushan ${ }^{5}$, Ana I. Silva (10) ${ }^{6,7}$, Marianne B. M. van den Bree (10) ${ }^{7,8,9}$, David E. J. Linden ${ }^{6,7,9}$, Michael J. Owen (10) ${ }^{7,8}$, Jeremy Hall (10) ${ }^{7,8,9}$, Sarah Lippé ${ }^{2}$, Mallar Chakravarty ${ }^{10}$, Danilo Bzdok ${ }^{11,12}$, Carrie E. Bearden $\mathbb{D}^{5}$, Bogdan Draganski (i] ${ }^{1,13,75}$ and Sébastien Jacquemont $\mathbb{1}^{2,75 \bowtie}$

(c) The Author(s) 2021

Many copy number variants (CNVs) confer risk for the same range of neurodevelopmental symptoms and psychiatric conditions including autism and schizophrenia. Yet, to date neuroimaging studies have typically been carried out one mutation at a time, showing that CNVs have large effects on brain anatomy. Here, we aimed to characterize and quantify the distinct brain morphometry effects and latent dimensions across 8 neuropsychiatric CNVs. We analyzed T1-weighted MRI data from clinically and non-clinically ascertained CNV carriers (deletion/duplication) at the 1q21.1 $(n=39 / 28), 16 p 11.2(n=87 / 78), 22 q 11.2(n=75 / 30)$, and $15 q 11.2$ ( $n=72 / 76$ ) loci as well as 1296 non-carriers (controls). Case-control contrasts of all examined genomic loci demonstrated effects on brain anatomy, with deletions and duplications showing mirror effects at the global and regional levels. Although CNVs mainly showed distinct brain patterns, principal component analysis (PCA) loaded subsets of CNVs on two latent brain dimensions, which explained 32 and $29 \%$ of the variance of the 8 Cohen's d maps. The cingulate gyrus, insula, supplementary motor cortex, and cerebellum were identified by PCA and multi-view pattern learning as top regions contributing to latent dimension shared across subsets of CNVs. The large proportion of distinct CNV effects on brain morphology may explain the small neuroimaging effect sizes reported in polygenic psychiatric conditions. Nevertheless, latent gene brain morphology dimensions will help subgroup the rapidly expanding landscape of neuropsychiatric variants and dissect the heterogeneity of idiopathic conditions.

Translational Psychiatry (2021)11:399; https://doi.org/10.1038/s41398-021-01490-9

\section{INTRODUCTION}

Genomic copy number variants (CNVs) are deletions or duplications of DNA segments of more than 1000 base pairs. Rare CNVs with large effects have been associated with a range of often overlapping developmental psychiatric phenotypes and conditions, including autism spectrum disorder (ASD) and schizophrenia (SZ) [1-4]. A looming question in psychiatric genetics pertains to the underlying basis of polygenicity: How do different variants lead to risk for the same psychiatric condition?

Some of the most frequent risk factors for neuropsychiatric disorders identified in pediatric clinics include CNVs at the 22q11.2, $16 p 11.2,1 q 21.1$, and $15 q 11.2$ genomic loci $[5,6]$. They affect the dosage of $60,29,12$ and 4 genes, respectively [7-9]. The largest increases in risk for SZ have been documented for the $22 q 11.2$ deletion ( 30 to 40 -fold) followed by $16 \mathrm{p} 11.2$ duplication (10-fold), $1 \mathrm{q} 21.1$ deletion and 15q11.2 deletion (1.5-fold) [2]. ASD risk is highest for $16 \mathrm{p} 11.2$ deletions and duplications (10-fold) followed by 1q21.1 duplications and 22q11.2 duplications (3 to 4-fold) [1, 2, 1013]. The nature and specificity of CNV effects on cognitive and behavioral traits is an area of intense investigation. All CNVs studied to date affect cognition to varying degrees and a broad range of cognitive functions [14, 15]. A recent study found that the range of affected traits was broadly similar for 13 CNVs at 8 loci and specific genotypes accounted for a low proportion of phenotypic variance [3]. These variants are therefore opportunities to investigate brain phenotypes conferring high-risk for mental illness.

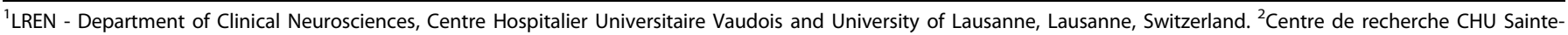

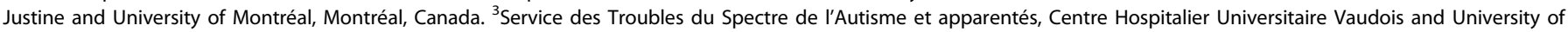
Lausanne, Lausanne, Switzerland. ${ }^{4}$ Applied Signal Processing Group (ASPG), Swiss Federal Institute Lausanne (EPFL), Lausanne, Switzerland. ${ }^{5}$ Semel Institute for Neuroscience and

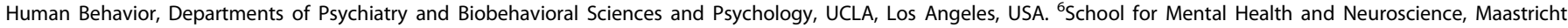
University, Maastricht, Netherlands. ${ }^{7}$ MRC Centre for Neuropsychiatric Genetics and Genomics, Cardiff University, Cardiff, UK. ${ }^{8}$ Division of Psychological Medicine and Clinical Neurosciences, School of Medicine, Cardiff University, Cardiff, UK. ${ }^{9}$ Neuroscience and Mental Health Research Institute, Cardiff University, Cardiff, UK. ${ }^{10}$ Douglas Research Centre,

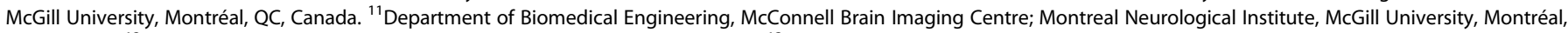

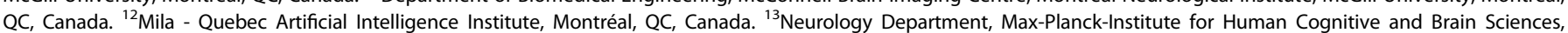
Leipzig, Germany. ${ }^{74}$ These authors contributed equally: Claudia Modenato, Kuldeep Kumar. ${ }^{75}$ These authors contributed equally: Bogdan Draganski, Sébastien Jacquemont. 凶email: sebastien.jacquemont@umontreal.ca
}

Received: 12 February 2021 Revised: 3 June 2021 Accepted: 16 June 2021

Published online: 20 July 2021 
Neuroimaging studies have only been performed for a few CNVs. Robust effects on total and regional brain volumes, cortical thickness (CT) and surface area (SA), have been reported in 22q11.2 [12, 13, 16], 16p11.2 BP4-5 [17-19], and 15q11.2 CNVs [20-23]. Opposing effects on global and-or regional brain volumes between deletions and duplications were observed for $16 \mathrm{p} 11.2$ [19], 22q11.2 [16], 1q21.1 [24] and 15q11.2 [20] loci (hereafter referred to as "mirror effects").

Neuroanatomical alterations associated with $16 \mathrm{p} 11.2$ and 22q11.2 show overlap with those observed in idiopathic ASD and SZ [17-19, 21, 25]. Finally, most of the effects are observed irrespective of psychiatric diagnoses and symptoms [12], suggesting that the final clinical outcome may result from the effect of CNVs and additional factors.

Neuroimaging studies across genomic variants are scarce. An investigation of 49 unaffected carriers of SZ-associated CNVs across five genomic loci in the UK biobank showed smaller volumes of the thalamus, hippocampus, and nucleus accumbens [26]. Functional connectivity similarities have also been demonstrated between $16 p 11.2$ and 22q11.2 deletions as well as with idiopathic ASD and SZ [27]. Alternatively, a recent study suggests a relatively distinct association between neuroimaging alterations and six different CNVs [28].

In this study, we aimed to characterize shared and distinct neuroanatomical alterations associated with eight CNVs at four genomic loci. We analyzed high-resolution structural brain scans from the largest multi-site dataset of CNV carriers $(n=484$, of which 87 have not yet been published) and controls $(n=1296)$ to date. Different approaches were implemented, from simple casecontrol contrasts to one-view and multi-view multivariate pattern learning $[29,30]$. First, we compared brain morphometry features associated with each deletion and duplication using univariate linear models. Second, we quantified the shared variation of brain morphometry associated with eight CNVs using principal component analysis (PCA). To complement this single-view approach, a multi-view pattern-learning algorithm was carried out for the joint analysis of genetic and morphometry brain data, to identify latent 'gene-morphometry dimensions' (canonical correlation analysis, CCA). Primary analyses were performed using VBM for consistency with previous studies [19]. In addition, we carried out the same multivariate analyses using freesurfer-derived cortical SA and thickness to ensure that shared variation was not limited to one neuroimaging modality or analytical pipeline.

\section{METHODS \\ Participants}

Deletions and duplications carriers' neuroimaging data included in the study were selected on the following breakpoints (hg 19): 16p11.2 (BP4-5, 29.6-30.2MB), 1q21.1 (Class I, 146.4-147.5MB \& II, 145.3-147.5MB), 22q11.2 (BPA-D, 18.8-21.7MB) and 15q11.2 (BP1-2, 22.8-23.0MB), together with control individuals not carrying any CNVs at these loci (Table 1, Supplementary Table 1 and supplementary materials). Signed consents were obtained from all participants or legal representatives prior to the investigation. Of note, data of 87 CNV carriers have never been published. Clinically ascertained CNV carriers were recruited as either probands referred for genetic testing, or as relatives. Controls were either noncarriers within the same families or individuals from the general population. We pooled data from five cohorts. CNVs from non-clinical populations were identified in the UK Biobank [31, 32].

\section{MRI data}

Details for methods and analyses are provided in Supplementary material and Supplementary Methods 1-8. Data sample included T1-weighted (T1w) images at $0.8-1 \mathrm{~mm}$ isotropic resolution across all sites. Population description is available in Table 1 and Supplementary Table 1.

\section{Data quality check}

All data included in the analysis were quality checked by the same researcher (CM). A total of 107 structural brain scans from carriers and controls were excluded from further analysis based on visual inspection that identified significant artifacts compromising accurate tissue classification and boundary detection (Supplementary materials).

\section{MRI data processing}

Data for Voxel-Based Morphometry were preprocessed and analyzed with SPM12 (http://www.fil.ion.ucl.ac.uk/spm/software/spm12/) [33-35] running under MATLAB R2018b (https://www.mathworks.com/products/ new_products/release2018b.html). For surface-based feature extraction, we used FreeSurfer 5.3.0 (http://surfer.nmr.mgh.harvard.edu [36,37,]). Quality control was performed using standardized ENIGMA quality control procedures (http://enigma.ini.usc.edu/protocols/imaging-protocols/).

\section{Statistical analysis for global brain measures}

Global brain aggregate measures (TIV, total gray matter (GM) volume, total $\mathrm{SA}$, and mean (T) were adjusted for age, age ${ }^{2}$, and sex as fixed effects and scanning site as random factor. Non-clinically ascertained subjects from the UKBB are on average 30 years older than the clinically ascertained subjects. Because of this age difference we used age matched control groups for univariate analysis. Global measure z-scores for each CNV for clinically and non-clinically ascertained CNVs were calculated using 331 and 965 controls, respectively. All statistical analyses were performed in R, version 3.4.4 (https://www.r-project.org/), or in MatlabR2018b.

\section{Voxel-based measures and statistical analyses}

We performed whole-brain voxel-based analysis testing for voxel-wise volume differences within the mass-univariate analysis framework implemented in SPM (Supplementary Method 4). Cohen's $d$ (i.e., effect size) [38] maps were obtained by converting SPM T-maps using the CAT12 toolbox for SPM (http://www.neuro.uni-jena.de/cat/).

\section{Surface-based measures and statistical analyses}

In parallel to VBM, we used surface-based GLM-based analysis to test differences in CT and SA (SurfStat toolbox [39]).

\section{Neuromorphometrics and Desikan parcellations}

Parcellation into regions of interest (ROls) was performed using neuromorphometric atlas (http://www.neuromorphometrics.com/) for GM volume (130 ROls excluding white matter ROls), and using Desikan parcellation [37] for FreeSurfer-derived CT and SA (68 ROIs).

\section{Comparison of ranked Cohen's $d$ maps across CNVs}

To adjust for the unequal power to detect alterations across different CNV groups, which have different sample and effect sizes, we ranked the Cohen's $d$ values of all voxels (/vertices) for each statistical maps (CNV versus controls contrast). We then tested for spatial overlap between maps across CNVs after thresholding the tails of the distribution at the 15th \& 85th quantiles. Dice index was calculated using publicly available Matlab scripts (https://github.com/rordenlab/spmScripts).

\section{Null hypothesis testing using spin permutations and label shuffling}

We used spin permutation and label shuffling $[40,41]$ to calculate empirical $p$ values for (1) the deletion and duplication convergence pattern and (2) the correlation/dice index between two maps.

\section{Quantifying shared variation across CNVs using principal components (PC)}

PCs were derived to quantify shared morphometry variation across CNVs. We used Cohen's $d$ values of 130 neuroanatomical GM regions (neuromorphometrics atlas) of eight CNVs as input-variables (z-scored Cohen's $d$ contrasts adjusted for total GM and nuisance variables; FactoMineR package in R). The variance explained (coefficient of determination, R-squared) for each CNV-associated Cohen's $d$ map by PCs was obtained by running a linear model $(\mathrm{Im})$ in R; with PC1 and PC2 as independent explanatory variables and the CNV Cohen's d map as a dependent variable. 
Table 1. Demographics.

\begin{tabular}{|c|c|c|c|c|c|c|c|c|}
\hline \multicolumn{9}{|c|}{ Clinical ascertainment } \\
\hline CNV loci & Copy number & Age mean (SD) & Male/Female & TIV mean (SD) & FSIQ mean (SD) & ASD & SCZ & Other diagnosis \\
\hline $1 \mathrm{q} 21.1$ & $\begin{array}{l}\text { Deletions } \\
N=29\end{array}$ & $29(18)$ & $11 / 18$ & $1.22(0.14)$ & $\begin{array}{l}90.85(21.75) \\
N=26\end{array}$ & 1 & - & 7 \\
\hline $16 p 11.2$ & $\begin{array}{l}\text { Deletions } \\
N=83\end{array}$ & $17(12)$ & $47 / 36$ & $1.54(0.17)$ & $\begin{array}{l}82.17(14.99 \\
N=64\end{array}$ & 13 & - & 36 \\
\hline \multirow[t]{2}{*}{$22 q 11.2$} & $\begin{array}{l}\text { Deletions } \\
N=74\end{array}$ & $16(8.6)$ & $35 / 39$ & $1.30(0.15)$ & $\begin{array}{l}77.42(13.51) \\
N=48\end{array}$ & 9 & 2 & 32 \\
\hline & $\begin{array}{l}\text { Duplication } \\
N=22\end{array}$ & $20(14.2)$ & $15 / 7$ & $1.47(0.16)$ & $\begin{array}{l}97.83(20.34) \\
N=12\end{array}$ & 2 & - & 8 \\
\hline \multicolumn{2}{|c|}{ Controls $N=331$} & $26(14.6)$ & $189 / 142$ & $1.46(0.15)$ & $\begin{array}{l}106.73 \\
N=224\end{array}$ & 1 & - & 23 \\
\hline $1 q 21.1$ & $\begin{array}{l}\text { Duplication } \\
N=9\end{array}$ & $60.6(7)$ & $2 / 7$ & $1.55(0.14)$ & $\begin{array}{l}0.2(1.3) \\
N=9\end{array}$ & - & - & - \\
\hline \multirow[t]{2}{*}{$15 q 11.2$} & $\begin{array}{l}\text { Deletions } \\
N=72\end{array}$ & $63.4(7.6)$ & $31 / 41$ & $1.54(0.15)$ & $\begin{array}{l}-0.3(0.9) \\
N=63\end{array}$ & - & - & 2 \\
\hline & $\begin{array}{l}\text { Duplication } \\
N=76\end{array}$ & $62.9(7.3)$ & $36 / 40$ & $1.49(0.15)$ & $\begin{array}{l}0(1.1) \\
N=71\end{array}$ & - & - & 6 \\
\hline \multirow[t]{2}{*}{$16 \mathrm{p} 11.2$} & $\begin{array}{l}\text { Deletion } \\
N=4\end{array}$ & $65.6(3.2)$ & $3 / 1$ & $1.56(0.13)$ & $\begin{array}{l}0.8(0.5) \\
N=2\end{array}$ & - & - & - \\
\hline & $\begin{array}{l}\text { Duplication } \\
N=4\end{array}$ & $69.3(2.1)$ & $1 / 3$ & $1.29(0.11)$ & $\begin{array}{l}-1.6(0.2) \\
N=4\end{array}$ & - & - & - \\
\hline
\end{tabular}

CNV copy number variant, SD standard deviation, TIV total intracranial volume, FSIQ full scale IQ, UKB FI UK Biobank fluid intelligence, ASD autism spectrum disorders, SCZ schizophrenia (including * ICD10 code F25.9 Schizoaffective disorder, unspecified).

CNV carriers and controls from the clinically ascertained group come from five different cohorts (Supplementary Table 1), while non-clinically ascertained participants were identified in the UK Biobank. 16p11.2 and 22q11.2 from the UKBB were not included in the VBM and SBM due to small sample size. Other diagnosis included: language disorder, major depressive disorder, posttraumatic stress disorder (PTSD), unspecified disruptive and impulse-control and conduct disorder, social anxiety disorder, social phobia disorder, speech sound disorder, moderate intellectual disability, specific learning disorder, gambling disorder, bipolar disorder, conduct disorder, attention deficit/hyperactivity disorder ADHD, Substance abuse disorder, global developmental delay, motor disorder, obsessive compulsive disorder, sleep disorder, Tourette's disorder, mood disorder, eating disorders, transient tic disorder, trichotillomania, pervasive developmental disorder NOS, specific phobia, body dysmorphic disorder, mathematics disorder, dysthymic disorder.

Jointly modeling of gene-morphology dimensions using CCA We re-purposed CCA to simultaneously model the shared and distinct impact of the CNVs in causing distributed alterations in brain morphometry (130 grey matter regions) $[29,30]$. This principled doublymultivariate approach, widely used in neuroimaging studies [29, 30], was performed to identify modes of coherent co-variation that jointly characterize how CNVs and patterns of regional volumes systematically co-occur across subjects. Henceforth, we refer to the ensuing modes of covariation as 'CCA dimensions' or 'gene-morphology dimensions'.

\section{RESULTS}

\section{CNV effects on global brain morphometry}

Deletions and duplications of each genomic loci showed opposing effects on one or more global metrics: TIV, total GM volume, total SA, or mean CT (Fig. 1, Supplementary Table 2). The directionality of global effects differed across loci (Fig. 1a-c). Effects on GM and SA were less pronounced once adjusted for TIV (Supplementary Fig. 1).

\section{Overlapping deletion effects on regional morphometry}

Whole-brain VBM analyses contrasting each deletion and duplication group with controls showed mostly distinct brain patterns across CNVs (Fig. 2a, c, e, Supplementary Table 3). To investigate potential overlap across the four genomic regions, we ranked Cohen's $d$ maps and overlapped voxels with similar rankings. Using a threshold for voxels with Cohen's $d<15$ th and $>85$ th percentiles separately (Fig. 3c, e, g, i), we observed significant overlap between deletions ( $p$ value SHUFFLE $<10 \mathrm{e}-4$, Fig. 3a). Volumes of the middle and anterior cingulate extending to the supplementary motor cortex and of the cerebellum were 
a.

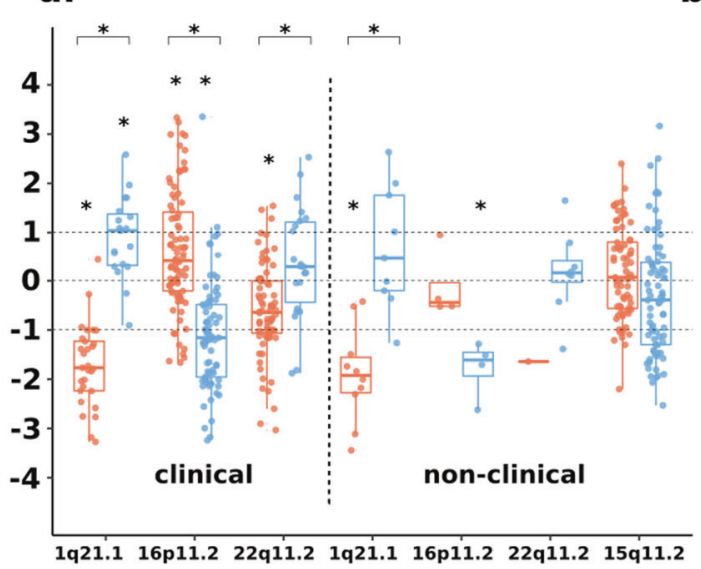

c. Total GM z-score

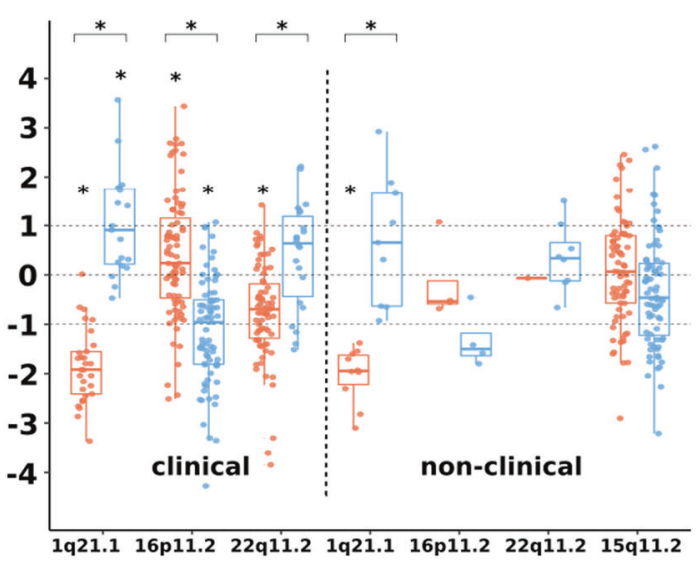

b.

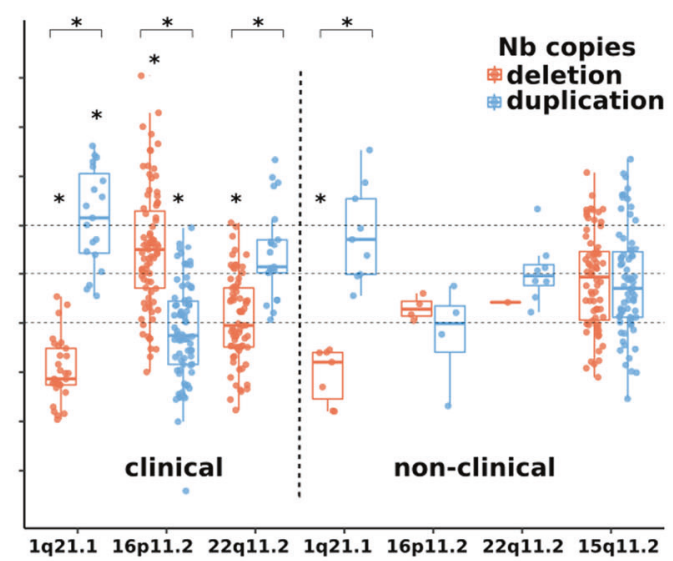

d.

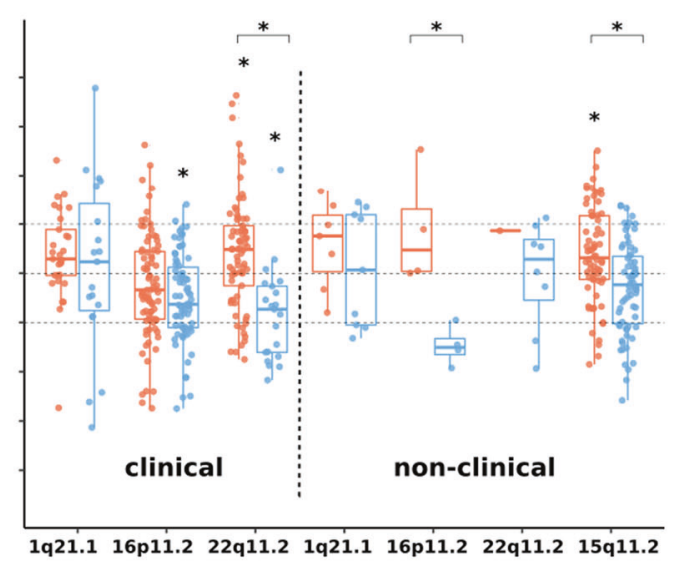

Fig. 1 1q21.1, 16p11.2, 22q11.2, and 15q11.2 exert rich effects on global brain measures. Total intracranial volume (a), total surface area (b), total grey matter volume (c) and mean cortical thickness (d) for clinically and non-clinically ascertained CNVs. $Z$ scores for clinically and non-clinically ascertained CNVs were calculated using 331 and 965 controls, respectively, adjusting for age, age ${ }^{2}$, sex and site as a random factor. $Y$ axis values are $Z$ scores. $X$ axis are CNV groups. Significant difference between CNV group and corresponding control group is indicated with a star. Horizontal bars with stars show significant differences between deletions and duplications within the same locus. TIV total intracranial volume, SA surface area, GM grey matter, CT cortical thickness.

decreased in all deletions while volume was increased in the thalamus (Fig. 3a).

Sensitivity analyses tested the effect of ascertainment and control groups: (1) We recomputed the deletion convergence map using 1q21.1 deletion carriers from UK Biobank instead of those clinically ascertained (Table 1). The new deletion convergence map was similar to the initial one presented above with a dice index of $39.4 \%$ ( $p$ value $_{\text {SPIN }}<10 \mathrm{e}-4$ ); (2) We excluded all subjects with autism, SZ, or other psychiatric diagnoses. Again, this did not change the overlap (Supplementary Fig. 2); (3) We tested the effects of the control group by recomputing contrasts only using controls from the same site (instead of the initial ANOVA pooling all controls together and controlling for site). This again did not alter the convergence maps (Supplementary Fig. 3). Finally, we performed the same analysis using Freesurfer-derived SA and CT measures. We also identified spatial overlaps but regions identified were different especially for CT (Supplementary Table 4 \& Fig. 4). Overlap maps are provided in Supplementary Figs. 5-8 and Tables 5, 6 .

Overlapping duplication effects on regional morphometry Contrasts computed for duplications (Fig. 2b, d, f) showed smaller effect sizes compared to deletions. The same analysis using
Cohen's $d$ values $<15$ th and $>85$ th percentiles (Fig. 3d, f, h, j) demonstrated spatial overlap across all four duplications ( $p$ value $_{\text {SHUFFLE }}<10 e-4$, Fig. 3b). The resulting pattern was mainly distinct from the one observed in deletions and was characterized by smaller volumes in anterior insula and frontal operculum, and larger volumes in the middle cingulate gyrus and supplementary motor cortex compared to controls.

Sensitivity analysis testing the effect of clinical ascertainment, psychiatric diagnoses, control groups, and volume versus Freesurfer-derived measures demonstrated that results were robust (Supplementary Figs. 2-8).

The deletion/duplication ratio of Cohen's $d$ distributions ranged from 1.24 to twofold across the four genomic loci ( $F$-test, $p<$ $10 \mathrm{e}^{-16}$, Fig. 3c-j, Supplementary Table 7). Similar effect-size ratios were also observed for SA alterations (Supplementary Table 7), except for the 15q11.2 locus.

We tested opposing (mirror) effects on VBM contrast maps between deletion and duplications. The strongest anticorrelation of Cohen's $d$ values was observed for $16 \mathrm{p} 11.2$ ( $p$ value SPIN $<10 \mathrm{e}$ $-4)$ followed by $15 q 11.2$ ( $p$ value $\left._{\text {SPIN }}<10 \mathrm{e}-4\right)$, 1 q21.1 ( $p$ value $<0.033$ ) and 22q11.2 ( $p$ value $_{\text {SPIN }}<0.038$ ) (Supplementary Fig. 9 and Tables 8-10). Mirror effects were observed in clinically and non-clinically ascertained CNV carriers, as well as for SA at all four 
a.

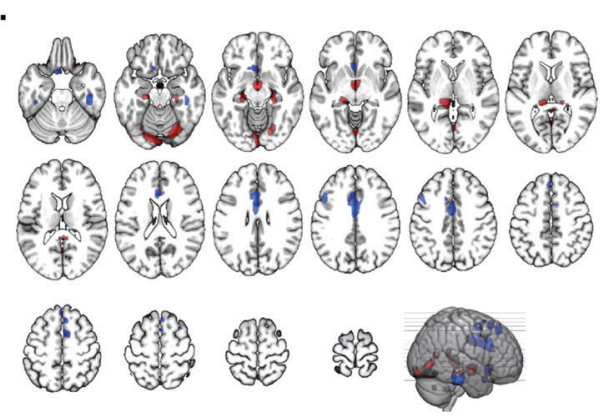

b.

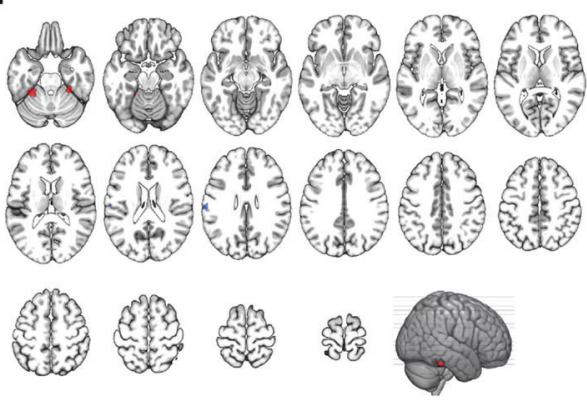

c.

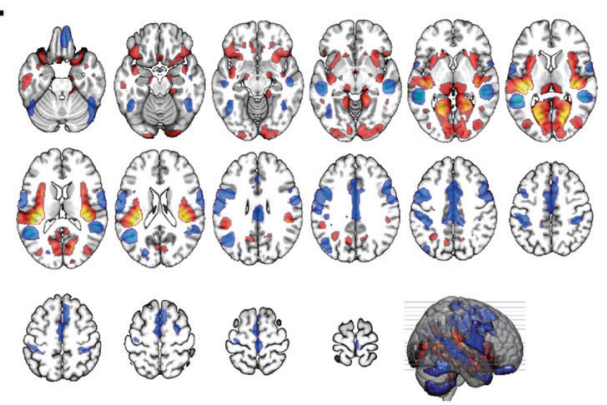

d.

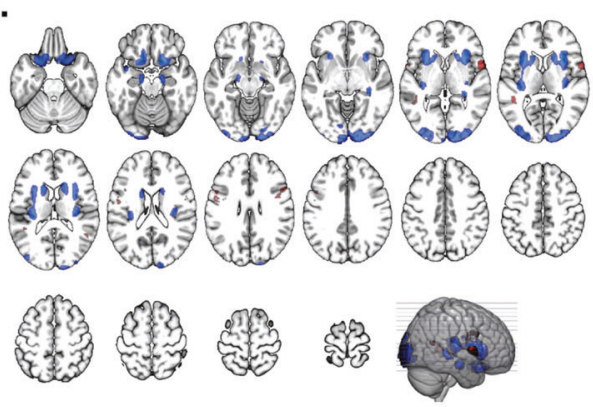

e.

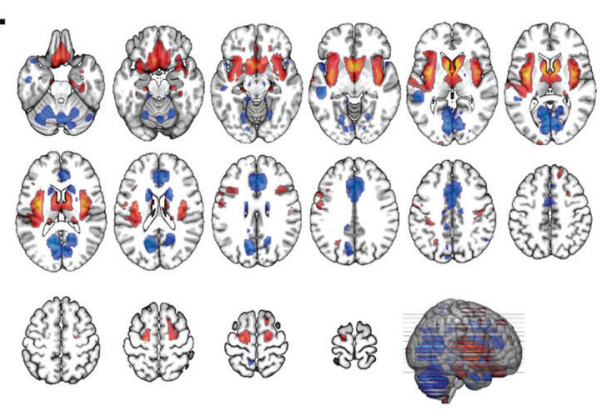

f.
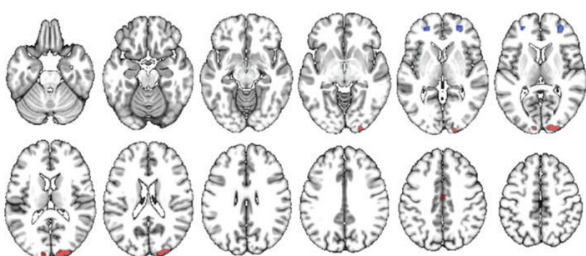

$22 q 11.2$

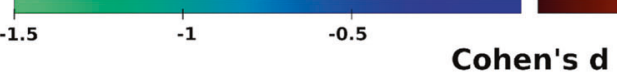

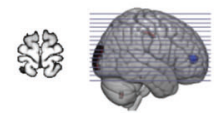

0.5

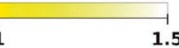

Fig. 2 Cohen's $d$ maps of VBM regional brain differences in deletion and duplication carriers at the 1q21.1, 16p11.2, and 22q11.2 loci compared to controls. Regional brain differences adjusted for total grey matter volume. Left and right columns show results for deletions (a, c, e) and duplication (b, d, f) carriers, respectively. Color maps show the significant effects of each CNV, thresholded at $q<0.05$ FWE. Color scale represents positive and negative Cohen's $d$ effect sizes were estimated. The linear model was adjusted for sex, linear, and quadratic expansion of age and total grey matter volume. 15q11.2 was not displayed because only a few voxels survived family-wise error (FWE) correction. Corresponding maps for surface area and cortical thickness are reported in Supplementary Figs. 4 and 5.

genomic loci but not for CT (Supplementary Tables 8-10). Hence mirror effects were observed in global metrics and, independently, in regional alterations.

\section{Quantifying distinct and shared effects on brain morphometry associated with eight CNVs}

We performed a multivariate PCA based on Cohen's $d$ profiles obtained from contrasts between the eight CNV groups and controls (using 130 neuromorphometric regional volumes, Supplementary Table 11). The first two components explained 31.8 and $28.7 \%$ of the variance of Cohen's $d$ maps, respectively. The third component dropped to $13.8 \%$ and was therefore not investigated further.

Deletions and duplications at each genomic loci showed opposite loading on PC1 or PC2 (Fig. 4C). Regions with the highest loadings on PC1 and PC2 were also those identified in the convergence maps presented above: in particular the middle cingulate gyrus and the supplementary motor cortex. Anterior and posterior insula, cerebellum, fusiform gyrus and thalamus were also top regions altered across subsets of CNVs (Fig. 4a, b and Supplementary Table 12). The variance explained by both components for each CNV's Cohen's $d$ map ranged from $27 \%$ to $82 \%$ (Fig. 4d). Finally, we performed the same analysis using Freesurfer-derived SA and CT measures which also provided latent dimensions with comparable variance explained, opposing loadings for deletions and duplications of each genomic loci (Supplementary Fig. 10). However, CNV loadings differ across brain morphometry metrics.

\section{Gene-morphology dimensions across eight CNVs}

As a next step, we performed a multi-view pattern-learning analysis, jointly analyzing the genetic and morphometry brain data. This doubly multivariate method allowed testing whether shared dimensions could be identified in a data-driven approach, without performing any individual contrast. We interrogated 2 hypotheses: (1) CNVs show levels of shared brain effects at the 
Spatial overlap Cohen's d tails

a.

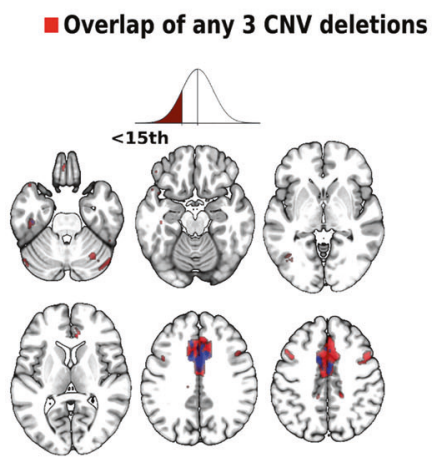

-
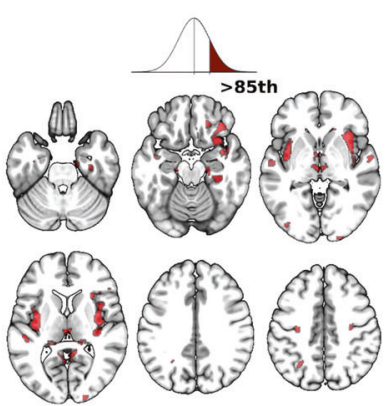

b.

- Overlap of any 3 CNV duplications

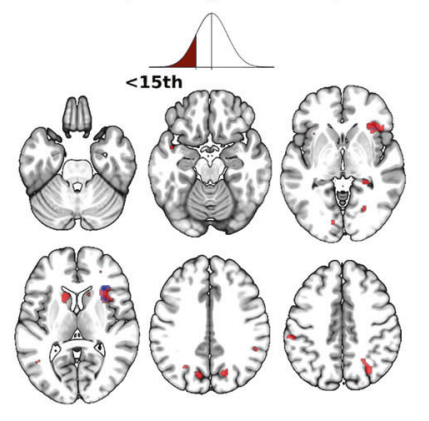

-Overlap of 4 CNV duplications

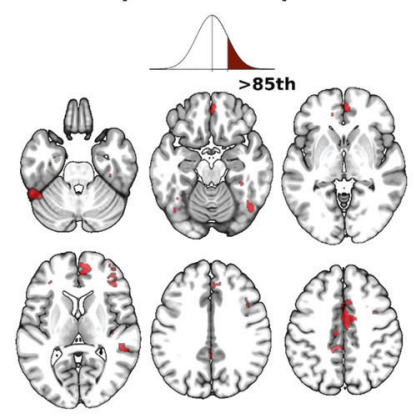

Distribution of Cohen's d

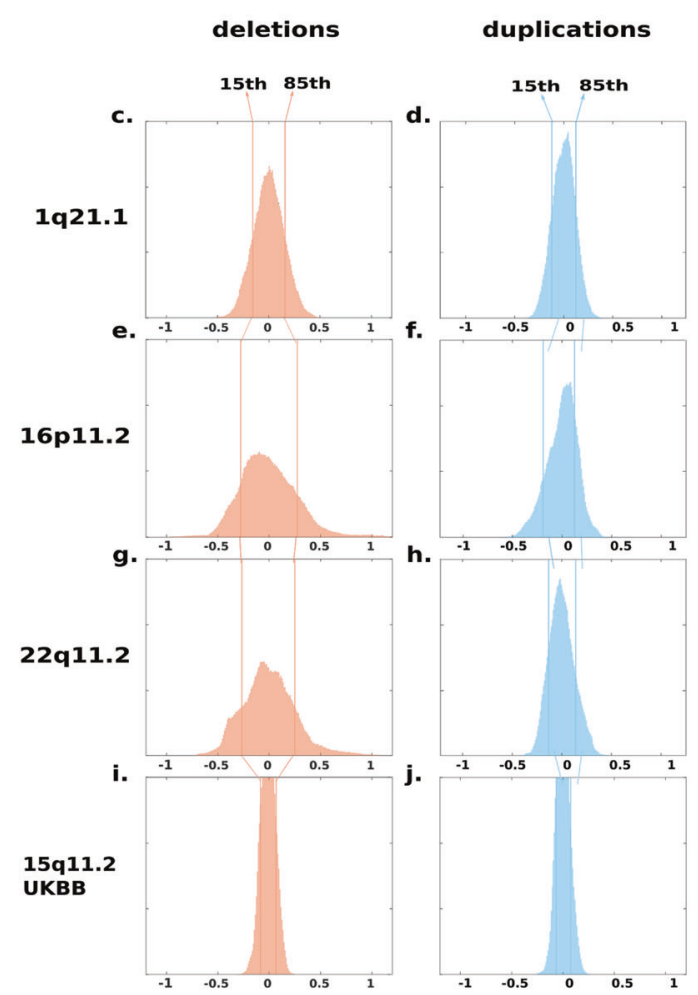

Fig. 3 Spatial overlap across deletions and duplications at four genomic loci. Spatial overlap across clinically and non-clinically ascertained deletions (a) and duplications (b) at four genomic loci shown separately for $<15$ th and $>85$ th percentile of Cohen's $d$ values. Overlap of all four deletions (a) or all four duplications (b) is shown in blue. Overlaps of any combination of three deletions (a) or any combination of three duplications (b) are shown in red. Top ranking Cohen's $d$ values used in $(\mathbf{a}, \mathbf{b})$ are presented on the density plots for all eight deletions and duplications: 1q21.1 (c, d), 16p11.2 (e, f), 22q11.2 (g, h), 15q11.2 (i, j). The $x$ axes values of the eight density plots are Cohen's $d$. Corresponding maps for surface area and cortical thickness are reported in Supplementary Figs. 6 and 7.

morphometry level and (2) deletions and duplications show opposing effects. We investigated the same 130 regional volumes in 484 carriers of CNVs at four genomic loci. To test hypothesis (2), deletions and duplications were coded as opposing gene dosage. CCA confirmed both hypotheses by identifying two significant 'gene-morphometry dimensions' ( $r=0.84,0.79, p$ value $<0.05$, Fig. $4 \mathrm{e}, \mathrm{f})$. Regional brain contributions to canonical dimension 1 and 2 were well correlated with those of PC2 and 1, respectively $(r=$ $0.83, r=-0.81$ ).

Top ranking brain regions contributing to either of the two CCA dimensions of morphological variation included supplementary motor cortex, posterior and anterior insula, middle cingulate gyrus, calcarine cortex, cuneus and accumbens (Supplementary Fig. 11 and Supplementary Table 13). 16p11.2 and 22q11.2 preferentially contributed to dimension 1 and 2 respectively, and 1q21.1 loaded similarly on both dimensions. 15q11.2 CNVs showed the smallest loadings on both dimensions (Fig. 4e).

Sensitivity analyses are detailed in supplementary material (Supplementary Figs. 12-16 and Tables 14, 15).

\section{DISCUSSION}

Here, in the largest cross-CNV-neuroimaging study to date, we tested potentially shared effects of eight neuropsychiatric CNVs on brain morphometry. CNVs showed a combination of distinct and shared profiles of brain alterations, as demonstrated by the spatial overlap of Cohen's $d$ maps across deletions and duplications. A multivariate approach (PCA) quantified distinct and shared alterations across subsets of CNVs and identified two latent dimensions explaining 31.8 and $28.7 \%$ of Cohen's $d$ map's variance. A second multivariate approach (CCA), jointly analyzing genetic and morphometry data, confirmed the latent CNV-brain dimensions identified by PCA. Genomic loci contributed to the latent CCA dimensions in proportion to their effect sizes. Even for small effect-size deletions at the 1q21.1 and 15q11.2 loci, the PCA components explained between 43 and $65 \%$ of their Cohen's $d$ profile. All three approaches-spatial overlap, CCA, and PCAidentified a similar set of regions altered by CNVs including the cingulate gyrus and supplementary motor cortex.

\section{Distinct and shared effects of CNVs}

Our results show that two-thirds of the average CNV effects on brain morphometry are distinct. This is consistent with a recent study showing relative specificity of association between brain patterns of gene expression and patterns of cortical anatomy changes across six CNVs and chromosomal aneuploidies [28]. Onethird of the effects on brain morphometry is shared as demonstrated by latent gene-morphology dimensions identified across subsets of CNVs. There is no single dimension explaining CNV effects. Instead, subsets of CNVs load on either dimension, which may suggest similar brain mechanisms within subgroups of CNV. Yet CNVs within subgroups were not characterized by the same risk for ASD or SCZ.

These results have implications for our conceptualization of polygenic psychiatric conditions. Indeed, studies estimate that $70-100 \%$ of any $1-\mathrm{MB}$ window in the human genome encompasses variants (including CNVs) contributing to increased risk for SZ and autism [4, 42]. Gene-morphology dimensions alone, can not explain the fact that subgroups of CNVs are associated with a similar range of behavioral symptoms [43], and psychiatric 
a.

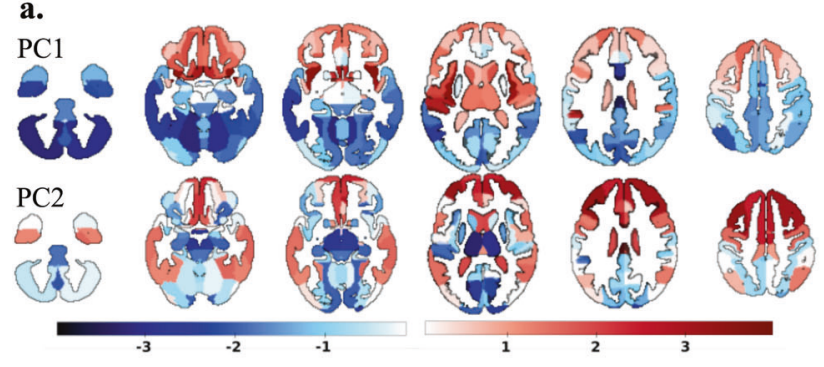

e.

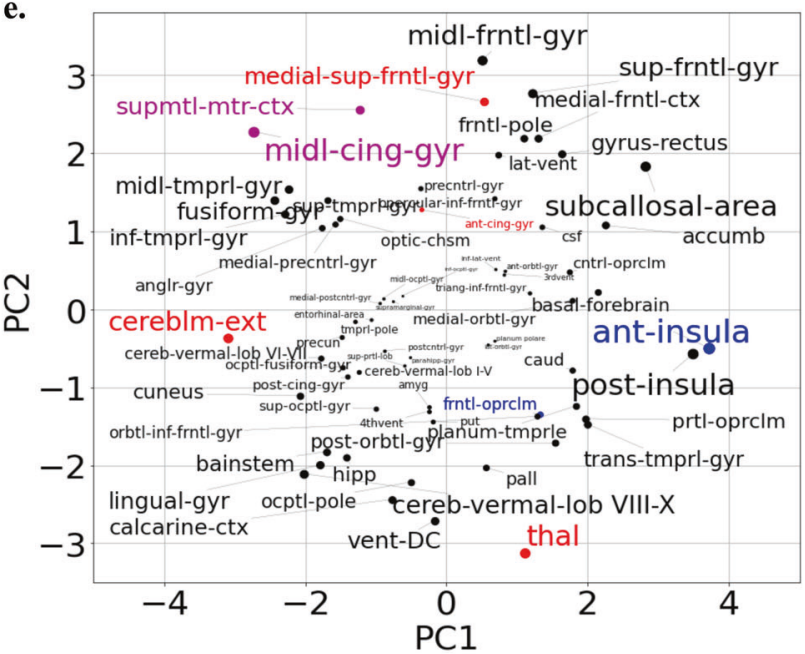

b.

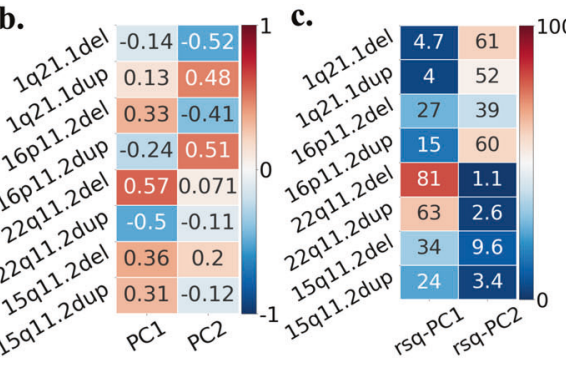

d.

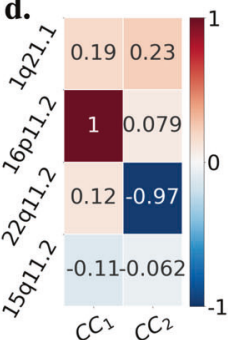

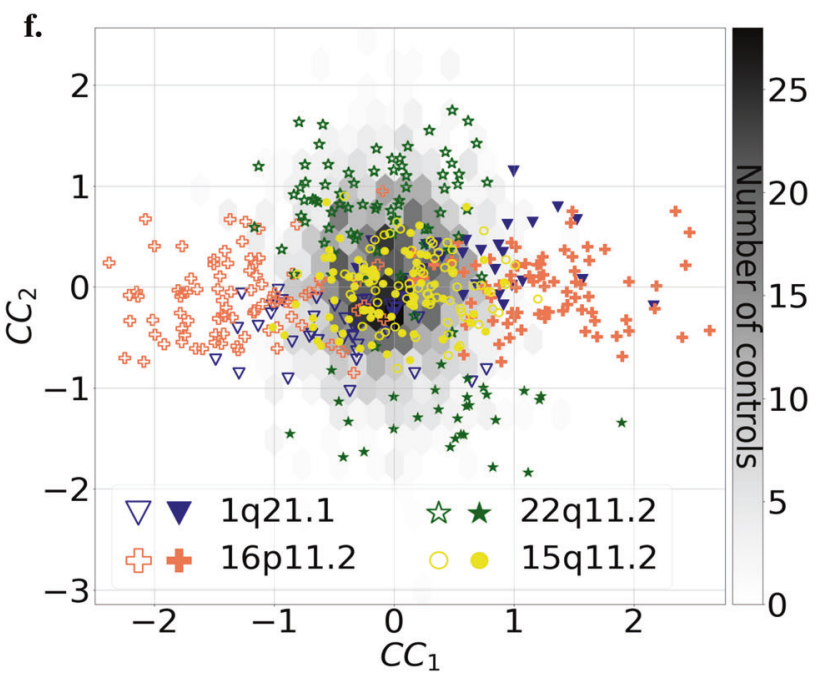

Fig. 4 Principal component analysis and canonical correlation analysis of brain alterations due to eight CNVs. a PCA dimension 1 and 2 regional relevances projected on axial brain slices. The darker the red or blue color, the stronger the positive or negative association with the PCA dimensions. PCA was run on z-scored Cohen's $d$ values, with the eight CNVs as variables and 130 neuroanatomical GM regions as observations. GM region volumes were adjusted for total grey matter, age, age ${ }^{2}$, sex, and site. The first two components explained respectively 31.77 and $28.66 \%$ of the variance. b Loading of eight CNVs on the two PCA dimensions. Values are PC loading magnitudes and represent the contribution of a CNV to the PC. c Variance explained (coefficient of determination, R-squared) of each CNV Cohen's $d$ profile by PC1 and PC2. Values and color scale represent the "percent of variance". $\mathbf{d}$ Loadings of the first and second CCA dimension on four CNV genomic loci. Shows contribution of a CNV loci to the canonical dimension. e Loading of Neuromorphometrics Regions of Interests (ROIs) on the two PCA dimensions. ROls are averaged across the left and right hemisphere for visualization. The font size is correlated to the region's contribution to dimensions. ROI names are color coded as being part of the deletion (red), duplication (blue) and both deletion and duplication (magenta) convergence patterns. f Scatterplot showing the participant/specific expressions of each of the 484 carriers of eight different CNVs along two dominant gene-morphometry canonical correlation (CC) dimensions established using 130 neuroanatomical GM regions of CNV carriers. GM region volumes were adjusted for total grey matter, age, age ${ }^{2}$, sex, and site. The empty and full symbols represent deletions and duplication, respectively. The grey hexagonal bin plot represents the frequency of controls $(n=1296)$. Controls were not used to calculate the CCA and were projected post hoc on the two dimensions using CCA prediction. CCA ROI loadings are reported in Supplementary Fig. 10. Results for surface area and cortical thickness are reported in Supplementary Fig. 9 (PCA), 14-15 (CCA).

disorders [1, 2, 4, 44]. In fact, the large proportion of distinct CNVneuroimaging effects suggests that a broad diversity of brain mechanisms increase the risk for autism and SZ. Extreme examples include CNVs associated with opposing loadings on the same latent gene-morphology dimension while increasing risk for the same psychiatric condition (ie. 16p11.2 deletions, duplications, and autism). The presence of such genomic variants in studies of ASD and SZ may explain heterogeneity and small neuroimaging effect sizes $[45,46]$. Why opposing effects on the same latent brain dimension increase risk for the same psychiatric condition is an unsolved question. Further observations on a broad variety of genomic variants are required to address this question.

\section{Brain hubs vulnerable to altered gene dosage}

Insula, cingulate, fusiform gyrus, and hippocampus are regions showing alterations across SZ, bipolar disorders, major depression, and obsessive-compulsive disorders [45, 47]. The cingulate, insula, and fusiform gyrus were also among regions markedly altered across eight CNVs. CNVs have either negative or positive effects on these brain regions, however, the number of CNVs included in this study did not allow us to associate the directionality of these effects with phenotypic traits. Alterations of the cingulate cortex have been associated with genetic and environmental risk for SZ [48]. The supplementary motor cortex has been shown to play a critical role in 16p11.2, 22q11.2 CNVs as well as autism and SZ by functional connectivity studies, but not by cross-diagnostic neuroimaging structural studies $[49,50]$. Several cerebellar regions (vermis lobule VIII-X and cerebellar cortex) are highly sensitive to CNVs, which may be due to the cerebellum's protracted development [51]. The cerebellum has either been excluded or not reported by cross-disorder structural neuroimaging studies, but volume alterations have been associated with autism and SZ separately $[52,53]$. Multiple genetic mouse models of autism, as well as Down Syndrome, also show abnormal cerebellar development [54]. The same level of spatial overlap was observed for SA and CT but implicated mostly distinct sets of brain regions. This is in line with the distinct genetic contributions previously demonstrated for these cortical metrics [55].

\section{Dissociation between global and regional effects}

Results suggest that global and local effects may be mechanistically unrelated. 1q21.1 deletions and duplications highlight the contrast between very large effects on global measures, with small 
regional effects once adjusted for total GM. Dissociation is also observed between the directionalities of global and regional effects: all deletions are associated with a smaller cingulate and supplementary motor cortex volume irrespective of their effect on TIV and GM. Animal studies have proposed mechanisms for global $[8,56]$, but not regional effects of CNVs.

\section{Limitations}

Multiple sites included in the study may have introduced noise, but previous studies have shown that site effects do not influence the neuroanatomical patterns associated with CNVs at the 16p11.2, 22q11.2, and 15q11.2 loci [12, 19, 23]. While shared variation could have been influenced by clinical ascertainment or psychiatric diagnoses, our sensitivity analyses showed that this is not the case. The effect of medication on CNVs brain alterations could not be investigated in the current study as medication information was not available for the whole dataset. We were underpowered to properly investigate potential sex-related effects of $1 q 21.1$ and $15 q 11.2$ on brain morphometry. Of note, previous neuroimaging studies of large $22 q 11.2$ and $16 \mathrm{p} 11.2$ samples were unable to identify any sex-related effects $[19,25]$.

$15 q 11.2$ deletions and duplications have small effect sizes and larger samples would improve the accuracy of the brain morphometry signature. Systematic analysis through the two most widespread computational neuroanatomy frameworks (voxel-based and surface-based) shows that effects could not be attributed to the processing pipeline. Extending our approach to the rapidly expanding number of rare genomic variants associated with psychiatric disorders is required to draw a robust conclusion on the distinct and shared effects of CNVs on brain structure.

\section{CONCLUSIONS}

The simultaneous analyses and comparisons of several genomic variants demonstrate distinct CNV-associated alteration profiles as well as shared latent gene-morphology dimensions relevant to subsets of CNVs. Large proportions of distinct effects may provide some answers to the small neuroimaging effect sizes reported in idiopathic psychiatric conditions. The mechanisms underlying the identified latent dimensions remain unknown and pathway convergence may occur early on at the transcriptome and protein level, or at later stages (i.e., brain architecture or behavior). The hotly debated omnigenic model postulates that convergence may occur at early stages due to highly interconnected cell regulatory networks [57]. These approaches may help subgroup genomic variants based on their morphometry signature and dissect the heterogeneity of psychiatric conditions.

\section{REFERENCES}

1. Sanders SJ, et al. Insights into autism spectrum disorder genomic architecture and biology from 71 risk loci. Neuron. 2015;87:1215-33.

2. Marshall CR, et al. Contribution of copy number variants to schizophrenia from a genome-wide study of 41,321 subjects. Nat Genet. 2017;49:27-35.

3. Chawner SJRA, et al. A genetics-first approach to dissecting the heterogeneity of autism: phenotypic comparison of autism risk copy number variants. AJP. 2021;178:77-86

4. Douard E, et al. Effect sizes of deletions and duplications on autism risk across the genome. AJP. 2020;178:87-98.

5. Moreno-De-Luca $D$, et al. Using large clinical data sets to infer pathogenicity for rare copy number variants in autism cohorts. Mol Psychiatry. 2013;18:1090-5.

6. Crawford K, et al. Medical consequences of pathogenic CNVs in adults: analysis of the UK Biobank. J Med Genet. 2019;56:131-8.

7. Mefford $\mathrm{HC}$, et al. Recurrent rearrangements of chromosome $1 \mathrm{q} 21.1$ and variable pediatric phenotypes. N Engl J Med. 2008;359:1685-99.

8. Golzio C, et al. KCTD13 is a major driver of mirrored neuroanatomical phenotypes associated with the 16p11.2 CNV. Nature. 2012;485:363-7.

9. Jonas RK, Montojo CA, Bearden CE. The 22q11.2 deletion syndrome as a window into complex neuropsychiatric disorders over the lifespan. Biol Psychiatry. 2014;75:351-60
10. Zufferey $\mathrm{F}$, et al. A $600 \mathrm{~kb}$ deletion syndrome at $16 \mathrm{p} 11.2$ leads to energy imbalance and neuropsychiatric disorders. J Med Genet. 2012;49:660-8.

11. D'Angelo $D$, et al. Defining the effect of the $16 \mathrm{p} 11.2$ duplication on cognition, behavior, and medical comorbidities. JAMA Psychiatry. 2016;73:20-30.

12. Sun $D$, et al. Large-scale mapping of cortical alterations in 22q11.2 deletion syndrome: Convergence with idiopathic psychosis and effects of deletion size. Mol Psychiatry. 2018:1-13. https://doi.org/10.1038/s41380-018-0078-5.

13. Niarchou $M$, et al. Psychiatric disorders in children with $16 \mathrm{p} 11.2$ deletion and duplication. Transl Psychiatry. 2019;9:1-8.

14. Moberg PJ, et al. Neurocognitive functioning in patients with $22 q 11.2$ deletion syndrome: a meta-analytic review. Behav Genet. 2018;48:259-70.

15. Gur RE, et al. Neurocognitive development in $22 q 11.2$ deletion syndrome: comparison with youth having developmental delay and medical comorbidities. Mol Psychiatry. 2014;19:1205-11.

16. Lin A, et al. Mapping 22q11.2 gene dosage effects on brain morphometry. J Neurosci. 2017;37:6183-99.

17. Maillard AM, et al. The $16 \mathrm{p} 11.2$ locus modulates brain structures common to autism, schizophrenia and obesity. Mol Psychiatry. 2015;20:140-7.

18. Qureshi AY, et al. Opposing brain differences in 16p11.2 deletion and duplication carriers. J. Neurosci. 2014;34:11199-211.

19. Martin-Brevet $S$, et al. Quantifying the effects of $16 \mathrm{p} 11.2$ copy number variants on brain structure: a multisite genetic-first study. Biol Psychiatry. 2018;84:253-64.

20. Stefansson $\mathrm{H}$, et al. CNVs conferring risk of autism or schizophrenia affect cognition in controls. Nature. 2014;505:361-6.

21. Silva Al, et al. Reciprocal White Matter Changes Associated With Copy Number Variation at 15q11.2 BP1-BP2: A Diffusion Tensor Imaging Study. Biological Psychiatry. 2019;85:563-72.

22. Ulfarsson $\mathrm{MO}$, et al. $15 \mathrm{q} 11.2 \mathrm{CNV}$ affects cognitive, structural and functional correlates of dyslexia and dyscalculia. Transl Psychiatry. 2017;7:e1109.

23. van der Meer $D$, et al. Association of copy number variation of the $15 q 11.2$ BP1BP2 Region With Cortical and Subcortical Morphology and Cognition. JAMA Psychiatry. 2019:1-11. https://doi.org/10.1001/jamapsychiatry.2019.3779.

24. Sønderby IE, et al. 1q21.1 distal copy number variants are associated with cerebral and cognitive alterations in humans. Transl Psychiatry. 2021;11:1-16.

25. Sun $D$, et al. Large-scale mapping of cortical alterations in 22q11.2 deletion syndrome: Convergence with idiopathic psychosis and effects of deletion size. Mol Psychiatry. 2018:1-13. https://doi.org/10.1038/s41380-018-0078-5.

26. Warland A, Kendall KM, Rees E, Kirov G, Caseras X. Schizophrenia-associated genomic copy number variants and subcortical brain volumes in the UK Biobank. Mol Psychiatry. 2020;25:854-62.

27. Moreau CA, et al. Mutations associated with neuropsychiatric conditions delineate functional brain connectivity dimensions contributing to autism and schizophrenia. Nat Commun. 2020;11:5272.

28. Seidlitz J, et al. Transcriptomic and cellular decoding of regional brain vulnerability to neurogenetic disorders. Nat Commun. 2020;11:3358.

29. Smith SM, et al. A positive-negative mode of population covariation links brain connectivity, demographics and behavior. Nat Neurosci. 2015;18:1565-7.

30. Wang $\mathrm{H}-\mathrm{T}$, et al. Finding the needle in high-dimensional haystack: a tutorial on canonical correlation analysis. Neurolmage. 2020.

31. Miller $\mathrm{KL}$, et al. Multimodal population brain imaging in the UK Biobank prospective epidemiological study. Nat Neurosci. 2016;19:1523-36.

32. Sudlow $C$, et al. UK biobank: an open access resource for identifying the causes of a wide range of complex diseases of middle and old age. PLoS Med. 2015;12: e1001779.

33. Ashburner J, Friston KJ. Unified segmentation. Neurolmage. 2005;26:839-51.

34. Lorio $S$, et al. New tissue priors for improved automated classification of subcortical brain structures on MRI. Neuroimage. 2016;130:157-66.

35. Ashburner J. A fast diffeomorphic image registration algorithm. Neurolmage. 2007:38:95-113.

36. Fischl B, Sereno MI, Dale AM. Cortical surface-based analysis: II: inflation, flattening, and a surface-based coordinate system. Neurolmage. 1999;9:195-207.

37. Desikan RS, et al. An automated labeling system for subdividing the human cerebral cortex on MRI scans into gyral based regions of interest. Neurolmage. 2006;31:968-80.

38. Cohen J. Statistical power analysis for the behavioral sciences. Psychology Press; 1988.

39. Worsley KJ, et al. SurfStat: a matlab toolbox for the statistical analysis of univariate and multivariate surface and volumetric data using linear mixed effects models and random field theory. Neuroimage. 2009. https://doi.org/10.1016/S1053-8119 (09)70882-1.

40. Alexander-Bloch A, et al. On testing for spatial correspondence between maps of human brain structure and function. Neuroimage. 2018;178:540-51.

41. Reardon PK, et al. Normative brain size variation and brain shape diversity in humans. Science. 2018;360:1222-7. 
42. Loh P-R, et al. Contrasting genetic architectures of schizophrenia and other complex diseases using fast variance-components analysis. Nat Genet. 2015;47:1385-92.

43. Huguet $G$, et al. Measuring and estimating the effect sizes of copy number variants on general intelligence in community-based samples. JAMA Psychiatry. 2018;75:447-57.

44. Chawner SJRA, et al. Genotype-phenotype associations in children with copy number variants associated with high neuropsychiatric risk in the UK (IMAGINEID): a case-control cohort study. The Lancet Psychiatry. 2019;6:493-505.

45. Opel $\mathrm{N}$, et al. Cross-disorder analysis of brain structural abnormalities in six major psychiatric disorders: a secondary analysis of mega- and meta-analytical findings from the ENIGMA Consortium. Biol Psychiatry. 2020. https://doi.org/10.1016/j. biopsych.2020.04.027.

46. Bedford SA, et al. Large-scale analyses of the relationship between sex, age and intelligence quotient heterogeneity and cortical morphometry in autism spectrum disorder. Mol Psychiatry. 2020;25:614-28.

47. Goodkind $M$, et al. Identification of a common neurobiological substrate for mental illness. JAMA Psychiatry. 2015;72:305-15.

48. Tost $H$, Champagne FA, Meyer-Lindenberg A. Environmental influence in the brain, human welfare and mental health. Nat Neurosci. 2015;18:1421-31.

49. Moreau C, et al. Neuropsychiatric mutations delineate functional brain connectivity dimensions contributing to autism and schizophrenia. 2019. https://doi. org/10.1101/862615.

50. Kebets V, et al. Somatosensory-motor dysconnectivity spans multiple transdiagnostic dimensions of psychopathology. Biol Psychiatry. 2019;86:779-91.

51. Sathyanesan A, et al. Emerging connections between cerebellar development, behavior, and complex brain disorders. Nat Rev Neurosci. 2019;20:298-313.

52. Moberget $\mathrm{T}$, et al. Cerebellar volume and cerebellocerebral structural covariance in schizophrenia: a multisite mega-analysis of 983 patients and 1349 healthy controls. Mol Psychiatry. 2018;23:1512-20.

53. Traut $\mathrm{N}$, et al. Cerebellar volume in autism: literature meta-analysis and analysis of the autism brain imaging data exchange cohort. Biol. Psychiatry. 2018;83:579-88.

54. Ellegood J, et al. Clustering autism: using neuroanatomical differences in 26 mouse models to gain insight into the heterogeneity. Mol Psychiatry. 2015;20:118-25.

55. Grasby KL. The genetic architecture of the human cerebral cortex. Science. 2020;367:6484

56. Richter $\mathrm{M}$, et al. Altered TAOK2 activity causes autism-related neurodevelopmental and cognitive abnormalities through RhoA signaling. Mol Psychiatry. 2019;24:1329-50.

57. Boyle EA, Li YI, Pritchard JK. An expanded view of complex traits: from polygenic to omnigenic. Cell. 2017;169:1177-86.

\section{AUTHOR CONTRIBUTIONS}

CMod, $\mathrm{KK}, \mathrm{BD}$, and SJ designed the study, analyzed imaging data, and drafted the manuscript. CMod and KK did all the preprocessing and analysis of neuroimaging data, DB provided scripts and mentored the CCA analysis. CMor, CEB, and DB contributed in result interpretation and in the editing of the manuscript. CMod, $A M$ AP, SR, and SM-B recruited and scanned participants in the 16p11.2 European Consortium. SL, COM, NY, PT, ED, FT-D, VC, ARC, FD recruited and scanned participants in the Brain Canada cohort. LK collected and provided the data for the UCLA cohort. DEJL, MJO, MBMVdB, JH, and AIS provided the data for the Cardiff cohort. All authors provided feedback on the manuscript.

\section{FUNDING}

This research was supported by Calcul Quebec (http://www.calculquebec.ca) and Compute Canada (http://www.computecanada.ca), the Brain Canada MultiInvestigator initiative, the Canadian Institutes of Health Research, CIHR_400528, The Institute of Data Valorization (IVADO) through the Canada First Research
Excellence Fund, Healthy Brains for Healthy Lives through the Canada First Research Excellence Fund. JS is a recipient of a Canada Research Chair in neurodevelopmental disorders, and a chair from the Jeanne et Jean Louis Levesque Foundation. The Cardiff CNV cohort was supported by the Wellcome Trust Strategic Award "DEFINE" and the National Centre for Mental Health with funds from Health and Care Research Wales (code 100202/Z/12/Z). The CHUV cohort was supported by the SNF (Maillard Anne, Project, PMPDP3 171331). Data from the UCLA cohort provided by CEB (participants with 22q11.2 deletions or duplications and controls) was supported through grants from the NIH (U54EB020403), NIMH (R01MH085953, R01MH100900, R03MH105808), and the Simons Foundation (SFARI Explorer Award). CMod was supported by the doc.mobility grant provided by the Swiss National Science Foundation (SNSF). KK was supported by The Institute of Data Valorization (IVADO) Postdoctoral Fellowship program, through the Canada First Research Excellence Fund. DB was supported by the Healthy Brains Healthy Lives initiative (Canada First Research Excellence fund), and by the CIFAR Artificial Intelligence Chairs program (Canada Institute for Advanced Research). BD is supported by the Swiss National Science Foundation (NCCR Synapsy, project grant numbers 32003B 135679, 32003B_159780, 324730_192755 and CRSK-3_190185), the Roger De Spoelberch and the Leenaards Foundations. We thank all of the families participating at the Simons Searchlight sites, as well as the Simons Searchlight Consortium. We appreciate obtaining access to imaging and phenotypic data on SFARI Base. Approved researchers can obtain the Simons Searchlight population dataset described in this study by applying at https://base.sfari.org. We are grateful to all families who participated in the 16p11.2 European Consortium.

\section{COMPETING INTERESTS}

MBMVdB reports grants from Takeda Pharmaceuticals, outside the submitted work All other authors reported no biomedical financial interests or potential conflicts of interest.

\section{ADDITIONAL INFORMATION}

Supplementary information The online version contains supplementary material available at https://doi.org/10.1038/s41398-021-01490-9.

Correspondence and requests for materials should be addressed to S.J.

Reprints and permission information is available at http://www.nature.com/ reprints

Publisher's note Springer Nature remains neutral with regard to jurisdictional claims in published maps and institutional affiliations.

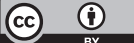

Open Access This article is licensed under a Creative Commons Attribution 4.0 International License, which permits use, sharing, adaptation, distribution and reproduction in any medium or format, as long as you give appropriate credit to the original author(s) and the source, provide a link to the Creative Commons license, and indicate if changes were made. The images or other third party material in this article are included in the article's Creative Commons license, unless indicated otherwise in a credit line to the material. If material is not included in the article's Creative Commons license and your intended use is not permitted by statutory regulation or exceeds the permitted use, you will need to obtain permission directly from the copyright holder. To view a copy of this license, visit http://creativecommons. org/licenses/by/4.0/.
\end{abstract}

(c) The Author(s) 2021

16P11.2 EUROPEAN CONSORTIUM

Marie-Claude Addor ${ }^{14}$, Joris Andrieux ${ }^{15}$, Benoît Arveiler ${ }^{16}$, Geneviève Baujat ${ }^{17}$, Frédérique Sloan-Béna ${ }^{18}$, Marco Belfiore ${ }^{19}$, Dominique Bonneau $^{20}$, Sonia Bouquillon ${ }^{21}$, Odile Boute ${ }^{22}$, Alfredo Brusco ${ }^{23}$, Tiffany Busa ${ }^{24}$, Jean- Hubert Caberg ${ }^{25}$, Dominique Campion ${ }^{26}$, Vanessa Colombert $^{27}$, Marie-Pierre Cordier ${ }^{28}$, Albert David ${ }^{29}$, François-Guillaume Debray ${ }^{30}$, Marie-Ange Delrue ${ }^{31}$, Martine Doco-Fenzy ${ }^{32}$, Ulrike Dunkhase-Heinl $^{33}$, Patrick Edery ${ }^{34}$, Christina Fagerberg ${ }^{35}$, Laurence Faivre ${ }^{36}$, Francesca Forzano ${ }^{37,38}$, David Genevieve ${ }^{39}$, Marion Gérard $^{40}$, Daniela Giachino ${ }^{41}$, Agnès Guichet ${ }^{42}$, Olivier Guillin ${ }^{43}$, Delphine Héron ${ }^{44}$, Bertrand Isidor ${ }^{45}$, Aurélia Jacquette ${ }^{46}$, Sylvie Jaillard $^{47}$, Hubert Journel ${ }^{48}$, Boris Keren ${ }^{49}$, Didier Lacombe ${ }^{50}$, Sébastien Lebon ${ }^{51}$, Cédric Le Caignec ${ }^{52}$, Marie-Pierre Lemaître ${ }^{53}$, James Lespinasse $^{54}$, Michèle Mathieu-Dramart ${ }^{55}$, Sandra Mercier ${ }^{56}$, Cyril Mignot ${ }^{57}$, Chantal Missirian ${ }^{58}$, Florence Petit ${ }^{59}$, Kristina Pilekær Sørensen ${ }^{60}$, Lucile Pinson ${ }^{61}$, Ghislaine Plessis ${ }^{62}$, Fabienne Prieur ${ }^{63}$, Alexandre Raymond ${ }^{64}$, Caroline Rooryck-Thambo ${ }^{65}$, Massimiliano 

Maldergem ${ }^{72}$

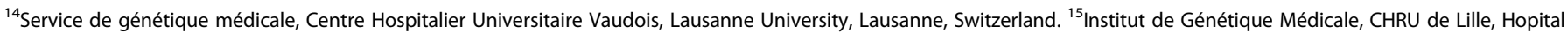

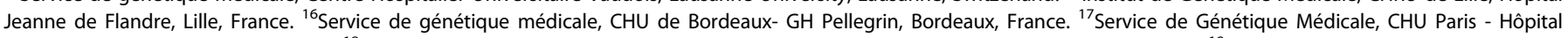

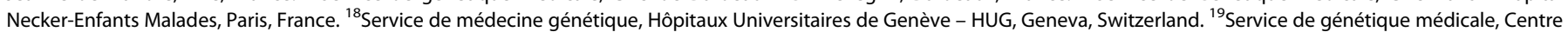

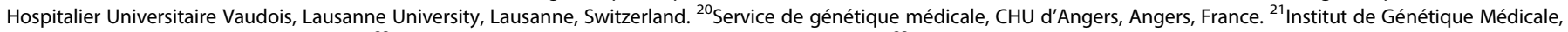

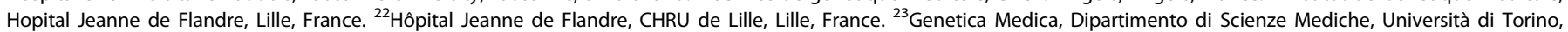

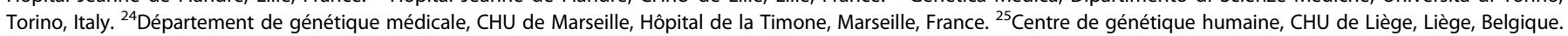

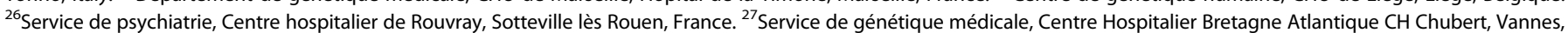

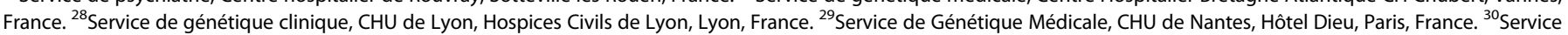

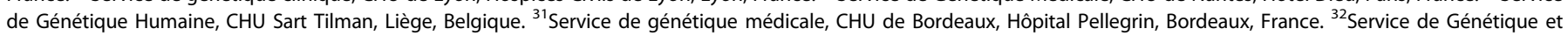

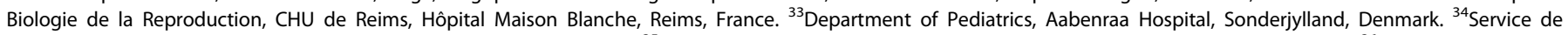

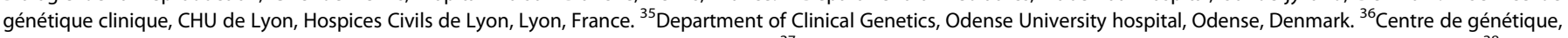

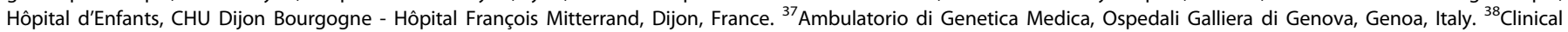

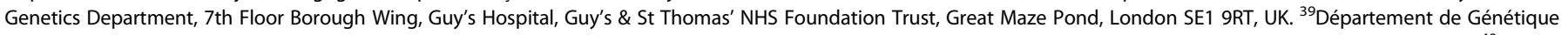

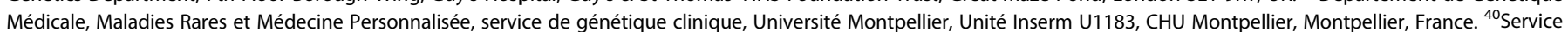

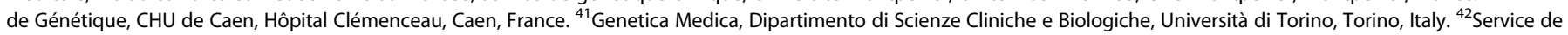

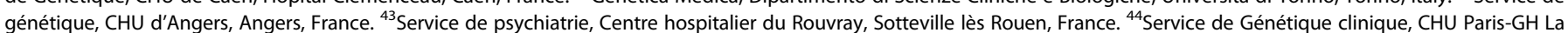

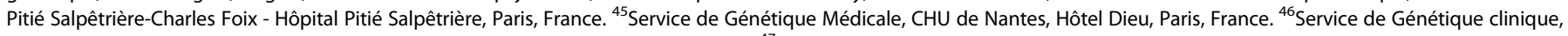

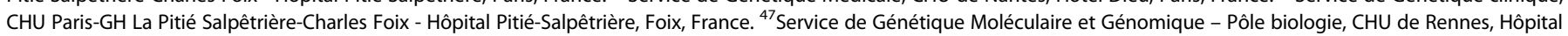

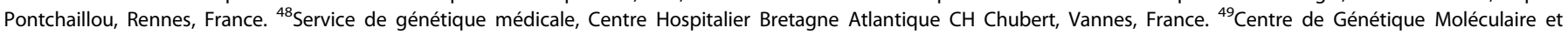

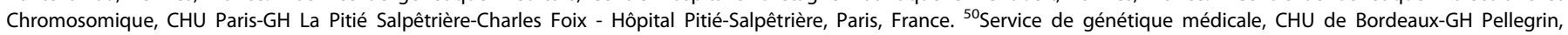

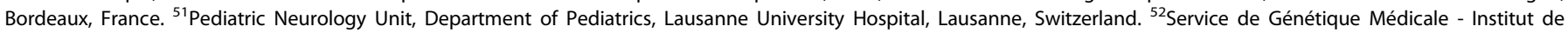

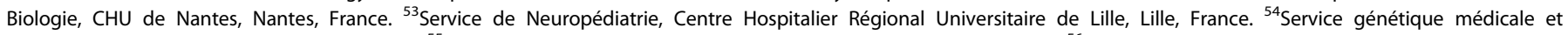

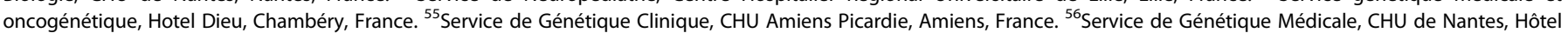

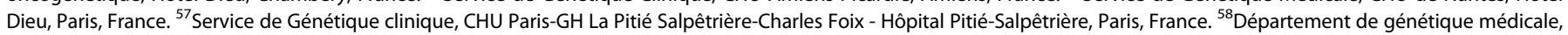

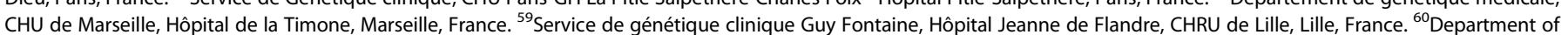

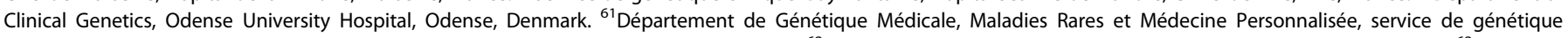

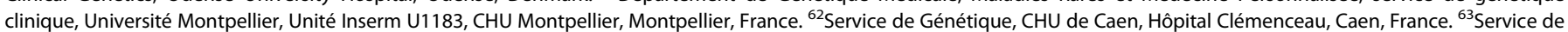

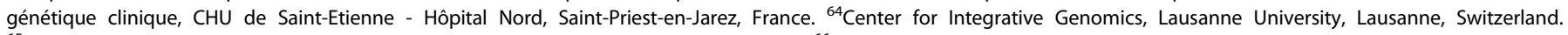

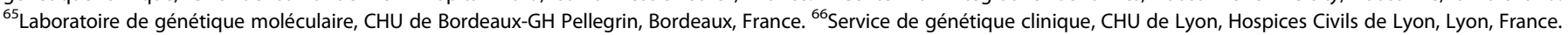

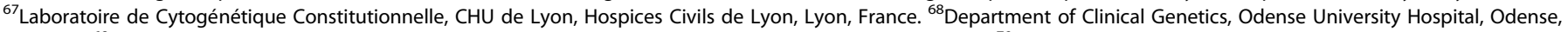

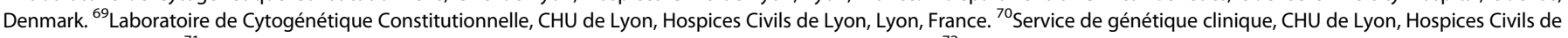

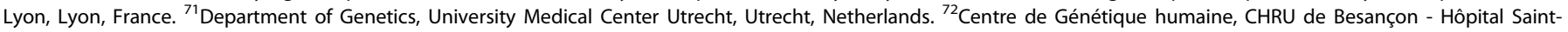
Jacques, Besançon, France
\end{abstract}

\title{
SIMONS SEARCHLIGHT CONSORTIUM
}

Hanalore Alupay ${ }^{73}$, Benjamin Aaronson ${ }^{73}$, Sean Ackerman ${ }^{73}$, Katy Ankenman ${ }^{73}$, Ayesha Anwar ${ }^{73}$, Constance Atwell ${ }^{73}$, Alexandra Bowe ${ }^{73}$, Arthur L. Beaudet ${ }^{73}$, Marta Benedetti ${ }^{73}$, Jessica Berg ${ }^{73}$, Jeffrey Berman ${ }^{73}$, Leandra N. Berry ${ }^{73}$, Audrey L. Bibb ${ }^{73}$, Lisa Blaskey ${ }^{73}$, Jonathan Brennan $^{73}$, Christie M. Brewton ${ }^{73}$, Randy Buckner ${ }^{73}$, Polina Bukshpun ${ }^{73}$, Jordan Burko ${ }^{73}$, Phil Cali ${ }^{73}$, Bettina Cerban ${ }^{73}$, Yishin Chang $^{73}$, Maxwell Cheong ${ }^{73}$, Vivian Chow ${ }^{73}$, Zili Chu ${ }^{73}$, Darina Chudnovskaya ${ }^{73}$, Lauren Cornew ${ }^{73}$, Corby Dale ${ }^{73}$, John Dell ${ }^{73}$, Allison G. Dempsey $^{73}$, Trent Deschamps ${ }^{73}$, Rachel Earl ${ }^{73}$, James Edgar ${ }^{73}$, Jenna Elgin ${ }^{73}$, Jennifer Endre Olson ${ }^{73}$, Yolanda L. Evans ${ }^{73}$, Anne Findlay ${ }^{73}$, Gerald D. Fischbach ${ }^{73}$, Charlie Fisk ${ }^{73}$, Brieana Fregeau ${ }^{73}$, Bill Gaetz ${ }^{73}$, Leah Gaetz ${ }^{73}$, Silvia Garza ${ }^{73}$, Jennifer Gerdts ${ }^{73}$, Orit Glenn ${ }^{73}$, Sarah E. Gobuty $^{73}$, Rachel Golembski ${ }^{73}$, Marion Greenup ${ }^{73}$, Kory Heiken ${ }^{73}$, Katherine Hines ${ }^{73}$, Leighton Hinkley ${ }^{73}$, Frank I. Jackson ${ }^{73}$, Julian JenkinsIII' ${ }^{73}$, Rita J. Jeremy ${ }^{73}$, Kelly Johnson ${ }^{73}$, Stephen M. Kanne ${ }^{73}$, Sudha Kessler ${ }^{73}$, Sarah Y. Khan ${ }^{73}$, Matthew Ku ${ }^{73}$, Emily Kuschner ${ }^{73}$, Anna L. Laakman ${ }^{73}$, Peter Lam ${ }^{73}$, Morgan W. Lasala ${ }^{73}$, Hana Lee ${ }^{73}$, Kevin LaGuerre ${ }^{73}$, Susan Levy ${ }^{73}$, Alyss Lian Cavanagh ${ }^{73}$, Ashlie V. Llorens $^{73}$, Katherine Loftus Campe ${ }^{73}$, Tracy L. Luks ${ }^{73}$, Elysa J. Marco ${ }^{73}$, Stephen Martin ${ }^{73}$, Alastair J. Martin ${ }^{73}$, Gabriela Marzano ${ }^{73}$, Christina Masson ${ }^{73}$, Kathleen E. McGovern ${ }^{73}$, Rebecca McNally Keehn ${ }^{73}$, David T. Miller ${ }^{73}$, Fiona K. Miller ${ }^{73}$, Timothy J. Moss ${ }^{73}$, Rebecca Murray $^{73}$, Srikantan S. Nagarajan ${ }^{73}$, Kerri P. Nowell ${ }^{73}$, Julia Owen ${ }^{73}$, Andrea M. Paal ${ }^{73}$, Alan Packer ${ }^{73}$, Patricia Z. Page ${ }^{73}$, Brianna M. Paul ${ }^{73}$, Alana Peters $^{73}$, Danica Peterson ${ }^{73}$, Annapurna Poduri ${ }^{73}$, Nicholas J. Pojman ${ }^{73}$, Ken Porche ${ }^{73}$, Monica B. Proud ${ }^{73}$, Saba Qasmieh ${ }^{73}$, Melissa B. Ramocki ${ }^{73}$, Beau Reilly ${ }^{73}$, Timothy P. L. Roberts ${ }^{73}$, Dennis Shaw ${ }^{73}$, Tuhin Sinha ${ }^{73}$, Bethanny Smith-Packard ${ }^{73}$, Anne Snow Gallagher ${ }^{73}$, Vivek Swarnakar $^{73}$, Tony Thieu ${ }^{73}$, Christina Triantafallou ${ }^{73}$, Roger Vaughan ${ }^{73}$, Mari Wakahiro ${ }^{73}$, Arianne Wallace ${ }^{73}$, Tracey Ward ${ }^{73}$, Julia Wenegrat $^{73}$ and Anne Wolken ${ }^{73}$

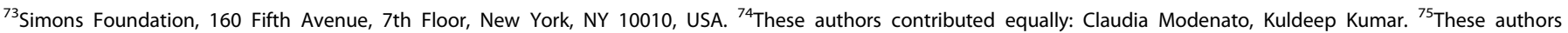
contributed equally: Bogdan Draganski, Sébastien Jacquemont. ${ }^{凶}$ email: sebastien.jacquemont@umontreal.ca
} 